\title{
Methodological Principles for the Introduction of Modality Categories in Modern Scientific Cognition*
}

\author{
Vitaly Ivlev \\ National Research University \\ Moscow State Technical University named after N.E. \\ Bauman (MSTU named after N.E.Bauman) \\ 5/1 2d Baumanskaya Street, Moscow, 105005, Russia \\ Federation \\ E-mail: vitalijivlev@yandex.ru
}

\author{
Mikhail Oseledchik \\ Department of philosophy and social sciences \\ High school of printing and media industry \\ Moscow Polytechnic University \\ 38 Bolshaya Semenovskaya Street, Moscow, 107023, \\ Russian Federation \\ E-mail: balu13@yandex.ru
}

\begin{abstract}
The article studies methodological principles of introducing necessity, randomness and chance categories in modern scientific cognition. The paper generalizes notions of different types of necessity, randomness and possibility and develops philosophical categories on this basis.
\end{abstract}

Keywords-philosophical categories; methodology; methodological principles; necessity; randomness; chance; modality; scientific cognition

\section{INTRODUCTION}

Modern philosophy devotes particular attention to the study of scientific philosophic foundations. These foundations include an ontological subsystem represented by a network of philosophic categories serving as a matrix for understanding and cognition of objects under study that are part of special scientific worldviews (physical, biological, etc.). One of the most important categories in this class are the categories of necessity, randomness and chance that have been used by scholars and philosophers since ancient times. The above mentioned categories are widely used in contemporary biological concepts as they are included in the system of biology's philosophical foundations. These foundations are an aggregate of philosophical ideas and principles that substantiate a biological worldview as well as biological references and standards [1]. The research paper discusses the possibility of introducing these philosophical categories into special sciences such as biology.

The article has the following objectives:

- to review methodological principles for the introduction of scientific notions;

- to identify typical descriptions of modality philosophical categories (necessity, chance, randomness);

- to develop methodological principles for the introduction of philosophical categories;

*The research has been carried out with funding from the Russian Humanitarian Science Foundation as part of a scientific research project Philosophical-methodological and natural scientific foundations of contemporary biological and ecological concepts) project № 16-23-01004
- to generalize the notion of different types of necessity, randomness and chance followed by the development of philosophical categories on their basis.

\section{METHODOLOGICAL PRINCIPLES FOR THE INTRODUCTION OF SCIENTIFIC NOTIONS}

Let us consider principles proposed by Yu.A. Petrov as methodological principles for the introduction of scientific notions $[2,3]$.

These principles include practicability principle of scientific notions introduction, compliance principle, efficacy principle, consistency principle and excludability principle. These principles are also called methodological conditions of scientific notions introduction. Les us describe the first three principles.

Practicability principle. This principle represents the following methodological requirement: it is practical to introduce a notion as a category of a certain science only when it is possible to develop this notion in the framework of this science and this notion is required to solve problems that science is facing. The first part of the requirement is called the condition of possibility while the last one is called the condition of necessary introduction.

Compliance principle: it is recommended to choose a type of definition that complies with the objectives met by this science. Yu.A.Petrov says: "This condition is fulfilled through the choice of definition type, level of its efficiency and accuracy that correspond to the optimum solution to the identified problems" [3, p. 24].

Yu.A.Petrov understands types of definitions as a definition itself and the explanation of terms called ostensive definition. E.K.Voishvillo and M.G.Degtyarev give the following characteristic to it: "...it specifies, at least, certain samples from the class of implicit subjects or the subject itself if we speak about a single subject" [4, p. 174].

As, according to logicians, there are several methods "similar to the definition", and most often in philosophy the definition of terms is substituted with their explanation through examples, it would be reasonable here to propose 
not the selection of a definition type but a solution to the problem instead: "To define or not to define a term?". If it is not defined, do we need to explain the term or not? If it is explained, then using which method? If the term is defined, then the logical type of definition should be selected (nominal or real, explicit or implicit).

The requirement "to choose a definition of certain efficiency" is an element of compliance principle.

A definition is efficient if it provides a method for identification of the defined objects. For instance, definitions of necessity and randomness categories are efficient to a certain extent. Properties and connections that are either the essence of this system or are determined by this essence are called required properties and connections. Then, random properties and connections in the system are properties and connections that are not essential and are not determined by the essence of this system.

These definitions point to a certain method for identification of the necessary and random. This method includes the following activities:

- to specify the system that has the aim to characterize its properties and connections under study as either necessary or random,

- to specify unique features of conditioning in this system,

- to reveal significant properties and connections in this system (i.e. the essence of the system),

- to specify if the property or connection under study is determined by the essence of this system. If the answer is positive, then this property or connection in this system will be necessary. If it is negative, then it will be random. [3, p. 28].

The third principle is efficacy of selected significant attributes of the introduced abstractions in terms of challenges that are met with their help. This principle may be formulated in the following manner: the content of the introduced scientific notions should include attributes that are substantial for the solution of scientific problems.

\section{TYPICAL DESCRIPTIONS OF MODALITY PHILOSOPHICAL CATEGORIES}

Let us now consider whether the above mentioned methodological principles are observed when modality categories are described (necessity, chance and randomness).

We will now study several typical cases of these categories' description.

In "The Notion" [5], "The Notion as a Form of Thinking" [6], "Logics as Part of Cognitive Theory and Scientific Methodology" [4] Voishvillo describes the notions of essence, significant and necessary attributed. All attributes of subjects belonging to a certain type are divided by E.K.Voishvillo into random and non-random. Random attributes are determined by subjects' external conditions while non-random attributes are not determined by external conditions. There are infinitely many non-random attributes of certain subjects (generic, non-random attributes). However, a person at some stage of cognition knows finitely many subject attributes of a certain type. All non-random attributes are significant. The last statement has not been explicitly expressed by E.K.Voishvillo, but it stems from the following text: "Some attributes determine others and the latter - the third attribute, etc. Due to this subordination, some system attributes may be characterized as more significant while others - as less significant" [6, p.118].

Thus, non-random, i.e. significant attributes, are those that are determined by internal conditions familiar to us and also by those that, according to our knowledge, are not determined by external conditions. Although, we do not know what kind of internal conditions determine them.

“Among subjects' non-random attributes, we can identify an aggregate of certain most significant (major) attributes that determine all the other attributes common for these subjects... An aggregate of major significant attributes inherent to a certain type of real specific subjects is called their essence" [6, p. 118-119]. And then: "The essence of a certain type of subjects is usually made up of attributes that are not available for observation. They are identified theoretically as a result of construction and substantiation of the theory that explains familiar attributes of subjects under study. Non-random attributes are separated from random attributes, first of all, in the course of such theoretical construction. Random attributes cannot be explained and do not fit into the system that appears through the construction of the theory" [6, p. 122].

While identifying significant attributes and significant derivative attributes (determined by major attributes), E.K. Voishvillo says that the last group can be characterized as "indispensably inherent" to subjects. The first group of attributes is inherent to subjects in practice and "in some trivial sense only - for the sake of generalization - we can speak about indispensable presence of these attributes as well..." [6, p. 122]. Thus, in fact, E.K. Voishvillo divides all attributes into random and non-random=significant and the latter - into main and derivative $=$ necessary. Main significant attributes are not necessary in the strict sense.

E.K. Voishvillo states that the main significant attributes are not necessary attributes in the exact sense of these characteristics and (they are not determined by other significant attributes) does not comply with his other assertions.

E.K. Voishvillo criticizes "the idea that a certain type of subjects or just certain subjects have a sort of absolute essence" which is the limit of these subjects' cognition [6,p. 119]. Then: "The essence of subjects having certain quality, i.e. subjects belonging to a certain class, revealed at a certain cognition level, is the main qualitative specifics of these subjects but only to the extent this specifics is familiar to us at this cognition stage. Considering this essence, we can explain their well-known attributes specific for these subjects (non-random). New qualities, properties of subjects that cannot be explained with the learnt essence are revealed amid further development of knowledge on the subjects. In 
this case, while looking for the required explanations, we penetrate into a deeper essence of subjects, discover attributes that can help explain all their peculiar features that have been previously learnt or newly discovered" [6, p. 120]. So, on the one hand, main significant attributes are not necessary in the exact sense of this word. On the other hand, in the end, they are determined by a deeper essence that we do not know yet, and are, thus, necessary.

What is the solution to this controversy? We should either admit that main significant attributes are necessary or allow a certain objectively existing limit to cognition.

Another problem discussed by E.K.Voishvillo is the following: can an aggregate of attributes be considered the essence or only laws belong to it? He says: "Laws...the core of the relationship determined by a certain essence... The essence of a certain class of subjects determines properties that are inherent to these subjects. Such dependence of phenomena is the law" [6, p.124].

It turns out that subjects' functioning laws are not included in their essence but represent interconnections of their essence and significant attributes not included in the essence. The laws themselves apparently should be qualified as necessary connections as they are determined by the essence.

The division of significant attributes into irrespectively significant and respectively significant is worth noticing. When criticizing Vvedensky, E.K.Voishvillo says: "Vvedensky does not make a distinction here between the two notions of the significant, specifically, the notion of attributes that are significant for subjects and attributes that are significant only in some relations (of the subject with other subjects) or from a certain point of view (a certain usage by a person)" [6, p. 128].

E.K.Voishvillo's concept is considered as most coherent. However, its research ends with the following questions: "Is it correct to consider all attributes belonging to a certain type of subjects determined by internal circumstances as significant?"; “Are main significant attributes necessary?"; "Can connections (laws) be characterized as significant or these characteristics can only be applied to attributes?".

\section{METHODOLOGICAL PRINCIPLES OF INTRODUCING PHILOSOPHICAL CATEGORIES}

As methodological principles are being discussed, it would be reasonable to develop the notion of methodology. Methodology are the guidelines that represent a set of prescriptions which can be used to reach the desired cognitive and practical result most efficiently (in terms of efforts, means, time spent). Methodological principles are most general prescriptions.

Practicability principle. As has been said before, Yu.A.Petrov identifies two parts in this principle that are called the conditions of necessity and possibility of introducing notions.

To our mind, the condition of necessity should be used as a guideline in scientific cognition which means developing notions including philosophical categories only when these notions are necessary for the solution of certain problems that this science is facing. The condition of possibility (it is reasonable to introduce a notion as a scientific category only when there is a possibility to develop this notion in the framework of science) is not obligatory.

First of all, if science does not have means to create a notion, then it would be impossible to create it.

Secondly, notions can be introduced when subjects making up the volume of notion are hypothetical. The notion of inheritable units, germs, and genes were introduced by G.Mendel following this principle. In his experiments, Mendel considered organisms as kinds of "black boxes". He did not know how genes looked like but, nevertheless, he managed to formulate laws describing inheritance mechanism.

Thus, instead of the efficacy principle, we formulate the principle of scientific notion necessary introduction: it is recommended to introduce a scientific notion only when this notion is required, together with other notions, to solve some problems that science is facing.

Compliance principle: a type of definition (including ostensive definition) that is used to introduce a term needs to comply with objectives met by science.

While implementing this principle, we solve the problem of whether we should limit ourselves to an ostensive definition or resort to one's own definition, and, secondly, in terms of the definition itself, we decide how effective the definition should be.

The first part of this principle should be explained the following way: when a scientific term is introduced, also into philosophy, it is necessary to decide whether to introduce a term through definition or using a method similar to definition (ostensive definition, description, characteristics, explanation with examples, comparison); if it is decided to introduce a term through definition, then a type of definition should be selected (nominal or real, explicit or implicit).

The first part of compliance principle that has been described is referred to general methodology and, apparently, relates rather to logics [7] than to theoretical philosophy or methodology and philosophy of science. That is why, instead of the efficiency principle, we can introduce its second part as an independent principle naming it efficiency principle: scientific notions need to be introduced based on efficient definitions. In this regard, the most efficient notions are revealed. Knowing the content of these efficient notions, one can construct or identify objects that relate to the volumes of these notions while inefficient notions do not possess the property. A typical trait of unscientific philosophy is incompliance with efficacy principle.

Principle of efficacy in the selection of significant attributes: content of the introduced notions should include attributes that are significant for the solution of scientific problems.

This principle is obligatory for mathematical and natural sciences, but, in philosophy, it is advisory. 
The above mentioned efficacy and compliance principles are logical or belong to general methodology and are not being reviewed in the paper.

Thus, when describing philosophical categories, it is recommended to use principles of necessary introduction, efficiency and, if possible, efficacy principle for the selection of significant attributes. Moreover, it is important to use a logical doctrine of the notion, rules and ways of terms definitions as well as methods similar to definition.

\section{TyPES OF NECESSITY, RANDOMNESS AND CHANCE}

Let us develop general generic and specific notions of necessity, randomness and chance [8].

At first, we will introduce the most general generic notion of necessity. Necessity is something that is determined by internal factors of an object, system, etc. or by external conditions or their existence. The utilized notion of definite determination can be explained through examples. Electrical conductivity of metals is determined by the presence of unbound electrons in them, and some diseases are not determined by genetic or chromosomal abnormalities; a disease can either occur or not occur with this kind of abnormalities which depends on circumstances. Indefinite determination exists objectively not only in biology but also in other sciences, at least, in natural sciences. In definite determination, a corresponding reason is a sufficient condition for the generation of a certain consequence. In indefinite determination (quasi determination), the reason is a sufficient condition for the generation of one certain consequence out of several consequences. However, it is actually impossible to identify which one.

The synonym to the necessity category as applied to future events is the word "imminence". Understanding of a necessity as imminence complies with both everyday and scientific usage of the "necessity" notion.

Let us shift from the general generic notion of necessity to specific notions. All the above mentioned notions of necessity (necessity as understood by Democritus and the understanding of necessity in modern biology) turn out to be specific in their relation to this general specific notion of necessity.

We will now formulate the following specific notions of necessity.

Classical (essential) necessity - something that is strictly determined by the essence of the object, system, etc. Genetic code of an organism is one of the examples illustrating essence. For instance, at least in some cases, the knowledge of organism's genetic code does not require the search of the organism's deeper essence.

Functional necessity: an attribute is necessary if conditions of its carrier's existence definitely determine the execution of certain functions by the attribute carrier. The notion of necessity not by origin that is utilized in biology can be one of the examples illustrating this notion. An attribute necessary for organisms to survive is an attribute that is preserved as determined by the internal essence of the system (population) that is considered together with the habitat.

Necessity by circumstances is a phenomenon that exists or is definitely determined by external conditions. This notion can be applied to social phenomena as well. Artificially created mutations, i.e. those created by direct influence on chromosomes and genes, can serve as one of the examples.

Let us now define the most general generic notion of randomness.

Randomness is something that is not determined by either internal factors of the object, system, etc. or by external conditions of their existence or it is determined but not definitely.

Let us formulate main specific notions of randomness.

Classical randomness is a phenomenon that is not definitely determined by the essence of the subject, the system.

Functional randomness: an attribute is random if conditions of its carrier's existence do not definitely determine or do not determine the execution of certain functions by the attribute carrier. Randomness not by origin described in the previous chapter can serve as an example of such randomness.

Randomness by circumstances is a phenomenon that exists or is not definitely determined by external conditions.

Finally, we will introduce the most general specific notion of chance. Chance is something the absence of which is not definitely determined either by internal factors or by external conditions.

Considering this understanding of chance, all the necessary turns out to be possible. Types of this general genetic notion of chance include chances that are quantitively characterized through the probability theory, i.e. when we take numbers (rational) less than zero and greater than unity as probability measures. Specific cases of chances in this approach are chances that generalize chances defined by Democritus:

- B1 - attribute inherent to the majority of subjects in the majority of cases;

- B2 - attribute inherent to the majority of subjects in the minority of cases;

- B3 - attribute inherent to the minority of subjects in the majority of cases;

- B4 - attribute inherent to half of subjects in half the cases;

- B5 - attribute inherent to the majority of subjects in the minority of cases.

Philosophical categories refer to scientific worldview (its philosophical foundations). Methodological principles as well as methods and ways of cognitive and practical activity are developed based on categories [9]. They serve as a 
reference in cognitive and practical activities and determine a certain approach to reality.

The knowledge of necessity, randomness and chance types facilitates the study of separate phenomena as it sets for the search of certain interconnections in a certain section of science. When phenomena that cannot be attributed to the described types of necessity, randomness or chance are revealed, the objective of science is to develop new notions. Then, these new notions will be used by philosophers to generalize notions that were created before and then the results of these generalizations will be used by specific sciences like, for example, modern biological concepts [10].

\section{CONCLUSION}

The article meets the objectives formulated in the beginning of the paper:

- methodological principles for the introduction of scientific notions were analyzed;

- typicaldescriptions of modality philosophical categories (necessity, chance, randomness) were identified;

- methodological principles for the introduction of philosophical categories were formulated;

- notions of different types of necessity, randomness and chance were generalized and corresponding philosophical categories were developed on its basis.

In the course of this research new problems that need to be solved were identified.

It is recommended to complement the research on the use of necessity, randomness and chance categories in modern biological concepts by the research into their utilization in ecological concepts.

It is recommended to study the development of the specified categories by modern philosophers and philosophers of the past in more detail $[11,12]$.

It is recommended to research the possibility of not using necessity, chance and randomness categories in scientific cognition. If such possibility exists, then it is advised to demonstrate the advantages of using the discussed categories or stop using them for the benefit of another category system which is less probable. Moreover, not only necessity, randomness and chance categories need to be specified but also other philosophical categories (essence, determinism, etc.). Such kind of work may result in further specification of modality philosophical categories.

\section{REFERENCES}

[1] Ivlev V.Yu., Ivleva M.L., Inozemtsev V.A., Udovik V.E. Informational society and formation of a new epistemological paradigm in modern science. Moscow, 2013.

[2] Petrov Yu.A. Methodological issues of scientific concepts implementation and development. Moscow, 1980.

[3] Petrov Yu.A., Nikiforov A.L. Logic and methodology of scientific cognition. Moscow, 1982.
[4] Voishvillo E.K., Degtyarev M.G. Logic as part of cognitive theory and scientific methodology. V. 2. Moscow, 1994

[5] Voishvillo E.K. The Notion. Moscow. 1967.

[6] Voishvillo E.K. The Notion as a Form of Thinking. Moscow, 1989

[7] Oseledchik M., Inozemtsev V., Ivlev V., Ivleva M. L. LogicalPhilosophical Approach to the Interpretation of the Concept of Knowledge //Proceedings of the 2016 3rd International Conference on Education, Language, Art and Inter-cultural Communication (ICELAIC 2016). Advances in Social Science, Education and Humanities Research. Paris: Atlantis Press, 2017. Volume 40. P.297301

[8] Ivlev V.Yu. Categories of necessity, chance and possibility: their meaning and methodological role in scientific knowledge. Philosophy and society. 1997. \# 5. P.108

[9] Inozemtsev V. A., Ivlev V. Yu., Ivleva M. L., Oseledchik M.B. Representology in the System of Modern Epistemology //Proceedings of the 2016 3rd International Conference on Education, Language, Art and Inter-cultural Communication (ICELAIC 2016). Advances in Social Science, Education and Humanities Research. Paris: Atlantis Press, 2017. Volume 40. P. 697-701

[10] Ivlev V. Yu., Ivleva M. L., Inozemtsev V. A., Oseledchik M.B. Categories of Modality and Their Use in Modern Biological Conceptions // Proceedings of the 2016 3rd International Conference on Education, Language, Art and Inter-cultural Communication (ICELAIC 2016). Advances in Social Science, Education and Humanities Research. Paris: Atlantis Press, 2017. Volume 40. P.687691

[11] Chistyakova O.V. Gnoseology of Orthodox Culture. Rostov on/Don. Monograph. 1998. P. 94. ISBN: 5-87872-023-X

[12] Chistyakova O.V. Russian Religious Philosophy as the Basis for State Identity // Journal of Eurasian Research. 2003. Volume 2. № 1. ISSN 1538-0378. P. 13-17. 